\title{
Gradient Ricci Soliton in Kenmotsu Manifold
}

\author{
Nirabhra Basu* and Arindam Bhattacharyya** \\ *Department of Mathematics, Bhawanipur Education Society College, Kolkata-700020, West Bengal, India, \\ **Department of Mathematics, Jadavpur University, Kolkata-700032,India,
}

\begin{abstract}
In this paper we have found that if a Kenmotsu manifold with a Killing vector field satisfies gradient Ricci soliton equation then the smooth function $f$ is either constant or $\nabla f$ is orthogonal to the Killing vector field.
\end{abstract}

Mathematics Subject classification 2000: Primary 53C44 ; Secondary 53D10, 53C25.

Keywords: Ricci flow, Ricci soliton, Kenmotsu manifold.

\section{Introduction}

In differential geometry, the Ricci flow is an intrinsic geometric flow, which was introduced by R. Hamilton [1]; [2] in 1982. But Ricci flow has got popularity after G. Perelman [3]; [4] used this to prove the Poincar'e conjecture and the sphere theorem.

The Ricci flow is a process that deforms the metric of a Riemannian manifold in a way formally analogous to the diffusion of heat, smoothing out irregularities in the metric. the Ricci flow equation is given by the following geometric evolution equation

$$
\frac{\partial g_{i j}}{\partial t}=-2 R_{i j} .
$$

Where $\mathrm{g}_{\mathrm{ij}}$ is Riemannian metric, $\mathrm{R}_{\mathrm{ij}}$ is Ricci tensor on the manifold $\mathrm{M}$ of dimension $\mathrm{n}$.

The concept of Ricci soliton was also introduced by R.S.Hamilton [5] in mid 80's. Ricci solitons[6] are natural generalizations of Einstein metrics. A Riemannian manifold (M, g) is called a Ricci soliton if there exits a smooth vector field $\mathrm{X}$ such that the Ricci tensor satisfies the following equation

$$
\text { Ric }+\frac{1}{2} £_{X} g=\lambda g,
$$

for some constant $\lambda$ and $E_{\mathrm{X}}$ is the Lie-derivative.

The Ricci soliton is called a gradient Ricci soliton[7] if $X=\nabla f$, for some smooth function $f$ on M. Ricci solitons are also correspond to self similar solutions of Hamilton's Ricci flow and often arise as limits of dilations of singularities in the Ricci flow. Presently lots of renowned scientists like H.D. Cao [8];[9], Chau [10], Chu [11], A.S. Dancer [12], Derdzinski [13], Ivey[14], Emineti, Fang, Feldman are working on Ricci flow.

As Ricci soltions are generalization of Einstein manifolds, one may look for new examples in the class where there are Einstein manifolds. A rich class of Einstein manifolds has been found in the class of Sasakian manifolds $[15,16,17,18,19,20,21]$. Chenxu and Meng [22] on their paper have shown that a Sasakian metric which also satisfies the gradient Ricci soliton equation is necessarily Einstein. Similar question aries in Kenmotsu manifold, i.e. does there exist a gradient Ricci soliton on Kenmotsu manifold which is not Einstein? We have tried to find out this in our paper.

\section{Preliminaries}

An $(2 m+1)$ dimensional smooth manifold $M$ is said to be an almost contact metric structure $(\phi, \xi, \eta, g)[23,24]$ consisting of a tensor field $\phi$ of type $(1,1)$, a vector field $\xi$, a 1 - form $\eta$ and a Riemannian metric g compatible with $(\phi, \xi, \eta)$ satisfying

$$
\begin{aligned}
& \phi^{2}=-\mathrm{I}+\eta \otimes \xi, \eta(\xi)=1, \phi(\xi)=0, \eta \circ \phi=0, \\
& \mathrm{~g}(\phi X, \phi Y)=g(X, Y)-\eta(X) \eta(Y) .
\end{aligned}
$$

An almost contact metric manifold is said to be Kenmotsu manifold $[25,26,27,28,29]$ if

$$
\left(\nabla_{X} \phi\right) Y=g(\phi X, Y) \xi-\eta(Y) \phi(X),
$$




$$
\nabla_{X} \xi=X-\eta(X) \xi .
$$

Where $\nabla$ denotes the Riemannian connection on $M$.

In Kenmotsu manifolds the following relations hold:

$$
\begin{array}{ll} 
& R(X, Y) Z=g(X, Z) Y-g(Y, Z) X, \\
& \left(\nabla_{X} \eta\right) Y=g(\phi X, \phi Y) \\
& \eta(R(X, Y) Z)=\eta(Y) g(X, Z)-\eta(X) g(Y, Z), \\
& R(X, Y) \xi=\eta(X) Y-\eta(Y) X, \\
& R(\xi, X) Y=\eta(Y) X-g(X, Y) \xi \\
\text { and } \quad & R(\xi, X) \xi=X-\eta(X) \xi .
\end{array}
$$

Now we shall prove a theorem on gradient Ricci soliton on Kenmotsu manifold.

\section{Result:}

Theorem: If $\left(\mathrm{M}^{2 \mathrm{~m}+1}, \mathrm{~g}\right)$ is a Kenmotsu manifold with Killing vector field $\xi$ satisfies the gradient Ricci soliton equation, then either $\mathrm{f}$ is a constant function i.e. (M,g) is an Einstein manifold or $\nabla f$ is orthogonal to $\xi$.

\section{Proof:}

From the equation (2.5), we get

$$
\mathrm{R}(\mathrm{Y}, \xi) \mathrm{Z}=\mathrm{g}(\mathrm{Y}, \mathrm{Z}) \xi-\mathrm{g}(\xi, \mathrm{Z}) \mathrm{Y}
$$

for vector fields $\mathrm{Y}, \mathrm{Z} \in \mathrm{TM}$.

Let $\mathrm{D} \subset \mathrm{TM}$, be the distribution defined by $\eta(Y)=g(Y, \xi)=0$, for $Y \in D$.

Now for $Y \in D$ and $Z \in T M$,we have

$$
\begin{aligned}
& \stackrel{R}{ }(\mathrm{Y}, \xi, \mathrm{Z}, \mathrm{Y})=\mathrm{g}(\mathrm{R}(\mathrm{Y}, \xi) \mathrm{Z}, \mathrm{Y}) \\
= & \eta(\mathrm{Y}) \mathrm{g}(\mathrm{Y}, \mathrm{Z})-\mathrm{g}(\mathrm{Z}, \xi)|\mathrm{Y}|^{2}
\end{aligned}
$$

$$
\text { i. e. }{ }^{\mathrm{R}}(\mathrm{Y}, \xi, \mathrm{Z}, \mathrm{Y})=-\mathrm{g}(\mathrm{Z}, \xi)|\mathrm{Y}|^{2} \text {. }
$$

Now if we take $Y=e_{i}$, where $e_{i}(i=1,2,3, \ldots, 2 m+1)$, is an orthonormal basis of the tangent space $T_{p} M$, we have

$$
\operatorname{Ric}(\xi, \mathrm{Z})=-2 \operatorname{mg}(\mathrm{Z}, \xi) .
$$

Again from (2.5), we have $\mathrm{R}(\mathrm{Y}, \xi) \xi=\mathrm{g}(\mathrm{Y}, \xi) \xi-\mathrm{g}(\xi, \xi) \mathrm{Y}$.

So, $g(R(Y, \xi) \xi, X)=g(Y, \xi) g(\xi, X)-g(\xi, \xi) g(Y, X)$. But as $\eta(Y)=g(Y, \xi)=0$, for $Y \in D$, we have

$$
` R(X, \xi, \xi, Y)=-g(X, Y) .
$$

Now if we take Lie derivative to the Ricci soliton equation(1.2), we get

$$
\left(E_{X} g\right)(\xi, W)=2(\lambda g(\xi, W)-\operatorname{Ric}(\xi, W)) .
$$

Using (3.3), and using the fact that $\mathrm{g}$ is a Riemainnian metric, we get after a brief calculation

$$
\mathrm{g}\left(\nabla_{\xi} X, W\right)+\mathrm{g}\left(\xi, \nabla_{\mathrm{W}} X\right)=2(\lambda+2 \mathrm{~m}) \mathrm{g}(\xi, \mathrm{W}) .
$$


Now if we put $\mathrm{W}=\xi$; we get

$$
\mathrm{g}\left(\nabla_{\xi} X, \xi\right)=\lambda+2 \mathrm{~m}
$$

Since $\left(\nabla_{\xi} g\right)(X, \xi)=0$, which implies $\nabla_{\xi} g(X, \xi)=g\left(\nabla_{\xi} X, \xi\right)+g\left(X, \nabla_{\xi} \xi\right)$.

Now considering $\xi$ as a Killing vector field, we have $\nabla_{\xi} \xi=0$.

$\Rightarrow \nabla_{\xi} \mathrm{g}(\mathrm{X}, \xi)=\mathrm{g}\left(\nabla_{\xi} \mathrm{X}, \xi\right)$.

Using this, (3.6) can be reduced to

$$
\nabla_{\xi} g(X, \xi)=\lambda+2 m \text {. }
$$

We know for a Killing vector field $\xi, €_{\xi}\left(£_{X} g\right)=0$.

$\Rightarrow\left(£_{\xi}\left(E_{\mathrm{X}} \mathrm{g}\right)\right)(\mathrm{Y}, \mathrm{Z})=0$, for $\mathrm{Y}, \mathrm{Z} \in \mathrm{TM}$.

Now using definition of Lie derivative and $g$ as a Riemannian metric we get

$$
\begin{gathered}
\nabla_{\xi}\left\{\mathrm{Xg}(\mathrm{Y}, \mathrm{Z})-\mathrm{g}\left(\nabla_{\mathrm{X}} \mathrm{Y}, \mathrm{Z}\right)-\mathrm{g}\left(\mathrm{Y}, \nabla_{\mathrm{X}} \mathrm{Z}\right)+\mathrm{g}\left(\nabla_{\mathrm{Y}} \mathrm{X}, \mathrm{Z}\right)+\mathrm{g}\left(\mathrm{Y}, \nabla_{\mathrm{Z}} \mathrm{X}\right)\right\}-\left\{\nabla_{\mathrm{X}} \mathrm{g}\left(\nabla_{\xi} \mathrm{Y}, \mathrm{Z}\right)\right. \\
-\nabla_{\mathrm{X}} \mathrm{g}\left(\nabla_{\mathrm{Y}} \xi, \mathrm{Z}\right)-\mathrm{g}\left(\nabla_{\mathrm{X}}\left(\nabla_{\mathrm{Y}} \xi-\nabla_{\xi} \mathrm{Y}\right), \mathrm{Z}\right)+\mathrm{g}\left(\nabla_{[\xi, \mathrm{Y}]} \mathrm{X}, \mathrm{Z}\right)-\mathrm{g}\left(\nabla_{\xi} \mathrm{Y}-\nabla_{\mathrm{Y}} \xi, \nabla_{\mathrm{X}} \mathrm{Z}\right. \\
\left.\left.-\nabla_{\mathrm{Z}} \mathrm{X}\right)\right\}-\left\{\nabla_{\mathrm{X}} \mathrm{g}\left(\mathrm{Y}, \nabla_{\xi} \mathrm{Z}-\nabla_{\mathrm{Z}} \xi\right)-\mathrm{g}\left(\nabla_{\mathrm{X}} \mathrm{Y}-\nabla_{\mathrm{Y}} \mathrm{X}, \nabla_{\xi} \mathrm{Z}-\nabla_{\mathrm{Z}} \xi\right)\right. \\
\left.-\mathrm{g}\left(\mathrm{Y}, \nabla_{\mathrm{X}}\left(\nabla_{\xi} \mathrm{Z}-\nabla_{\mathrm{Z}} \xi\right)\right)+\mathrm{g}\left(\mathrm{Y}, \nabla_{[\xi, \mathrm{Z}]} \mathrm{X}\right)\right\}=0 .
\end{gathered}
$$

Now as $\left(\nabla_{\mathrm{X}} \mathrm{g}\right)(\mathrm{Y}, \mathrm{Z})=\mathrm{Xg}(\mathrm{Y}, \mathrm{Z})-\mathrm{g}\left(\nabla_{\mathrm{X}} \mathrm{Y}, \mathrm{Z}\right)-\mathrm{g}\left(\mathrm{Y}, \nabla_{\mathrm{X}} \mathrm{Z}\right)=0$; we have

$$
\begin{array}{r}
\nabla_{\xi}\left(g\left(\nabla_{Y} X, Z\right)+g\left(\nabla_{Z} X, Y\right)\right)-\left\{\nabla_{X} g\left(\nabla_{\xi} Y, Z\right)-\nabla_{X} g\left(\nabla_{Y} \xi, Z\right)-g\left(\nabla_{X} \nabla_{Y} \xi, Z\right)\right. \\
+g\left(\nabla_{X} \nabla_{\xi} Y, Z\right)+g\left(\nabla_{[\xi, Y]} X, Z\right)-g\left(\nabla_{\xi} Y, \nabla_{X} Z\right)+g\left(\nabla_{\xi} Y, \nabla_{Z} X\right)+g\left(\nabla_{Y} \xi, \nabla_{X} Z\right) \\
\left.-g\left(\nabla_{Y} \xi, \nabla_{Z} X\right)\right\}-\left\{\nabla_{X} g\left(Y, \nabla_{\xi} Z\right)-\nabla_{X} g\left(Y, \nabla_{Z} \xi\right)-g\left(\nabla_{X} Y, \nabla_{\xi} Z\right)+g\left(\nabla_{X} Y, \nabla_{Z} \xi\right)\right. \\
+g\left(\nabla_{Y} X, \nabla_{\xi} Z\right)-g\left(\nabla_{Y} X, \nabla_{Z} \xi\right)-g\left(Y, \nabla_{X} \nabla_{\xi} Z\right)+g\left(Y, \nabla_{X} \nabla_{Z} \xi\right) \\
\left.+g\left(Y, \nabla_{[\xi, Z]} X\right)\right\}=0 .
\end{array}
$$

If we put $\mathrm{Z}=\xi$ and $\mathrm{Y} \in \mathrm{D}$ in the above equation, we get

$$
\begin{gathered}
\nabla_{\xi} g\left(\nabla_{Y} X, \xi\right)+\nabla_{\xi} g\left(\nabla_{\xi} X, Y\right)-\nabla_{X} g\left(\nabla_{\xi} Y, \xi\right)+\nabla_{X} g\left(\nabla_{Y} \xi, \xi\right)-g\left(\nabla_{X} \nabla_{Y} \xi, \xi\right)-g\left(\nabla_{X} \nabla_{\xi} Y, \xi\right) \\
=0
\end{gathered}
$$

In (3.9) we can put the following results :

$$
\begin{aligned}
& \text { (i) } \nabla_{\xi} g\left(\nabla_{Y} X, \xi\right)=g\left(\nabla_{\xi} \nabla_{Y} X, \xi\right) ; \\
& \text { (ii) } \nabla_{\xi} g\left(\nabla_{\xi} X, Y\right)=g\left(\nabla_{\xi} \nabla_{\xi} X, Y\right)+g\left(\nabla_{\xi} Y, \nabla_{X} \xi\right) ; \\
& \text { (iii) } \nabla_{X} g\left(\nabla_{\xi} Y, \xi\right)=g\left(\nabla_{X} \nabla_{\xi} Y, \xi\right)+g\left(\nabla_{\xi} Y, \nabla_{X} \xi\right) ; \\
& \text { (iv) } \nabla_{X} g\left(\nabla_{Y} \xi, \xi\right)=g\left(\nabla_{X} \nabla_{Y} \xi, \xi\right)+g\left(\nabla_{Y} \xi, \nabla_{X} \xi\right) .
\end{aligned}
$$


Using the above results in equation (3.9), it will reduce into the equation

${ }^{\prime} \mathrm{R}(\xi, Y, X, \xi)+\mathrm{g}\left(\nabla_{\mathrm{Y}} \nabla_{\xi} \mathrm{X}, \xi\right)+\mathrm{g}\left(\nabla_{\xi} \nabla_{\xi} \mathrm{X}, \mathrm{Y}\right)-2 \mathrm{~g}\left(\nabla_{\mathrm{X}} \nabla_{\xi} \mathrm{Y}, \xi\right)+\mathrm{g}\left(\nabla_{\mathrm{Y}} \xi, \nabla_{\xi} \mathrm{X}\right)=0$.

Now as $Y \in$ D, i.e. $\nabla_{\xi} Y=\nabla_{Y} \xi=0$, the equation reduces to

$$
\text { 'R }(X, \xi, \xi, Y)+g\left(\nabla_{\xi} \nabla_{\xi} X, Y\right)+g\left(\nabla_{Y} \nabla_{\xi} X, \xi\right)=0
$$

Also we have

$$
\left(\nabla_{Y} g\right)\left(\nabla_{\xi} X, \xi\right)=\nabla_{Y} g\left(\nabla_{\xi} X, \xi\right)-g\left(\nabla_{Y} \nabla_{\xi} X, \xi\right)-g\left(\nabla_{\xi} X, \nabla_{Y} \xi\right),
$$

which implies $g\left(\nabla_{Y} \nabla_{\xi} X, \xi\right)=\nabla_{Y} g\left(\nabla_{\xi} X, \xi\right)$.

Using this in (3.10) the equation becomes

$$
\mathrm{r}(\mathrm{X}, \xi, \xi, \mathrm{Y})+\mathrm{g}\left(\nabla_{\xi} \nabla_{\xi} \mathrm{X}, \mathrm{Y}\right)+\nabla_{\mathrm{Y}} \mathrm{g}\left(\nabla_{\xi} \mathrm{X}, \xi\right)=0 .
$$

From (3.6), we have $g\left(\nabla_{\xi} X, \xi\right)=\lambda+2 \mathrm{~m}$.

$\Rightarrow \nabla_{\mathrm{Y}} \mathrm{g}\left(\nabla_{\xi} \mathrm{X}, \xi\right)=0$.

So (3.11) becomes ${ }^{`} \mathrm{R}(\mathrm{X}, \xi, \xi, \mathrm{Y})+\mathrm{g}\left(\nabla_{\xi} \nabla_{\xi} \mathrm{X}, \mathrm{Y}\right)=0$.

Now using (3.4), we have

$$
\mathrm{g}\left(\nabla_{\xi} \nabla_{\xi} \mathrm{X}, \mathrm{Y}\right)=\mathrm{g}(\mathrm{X}, \mathrm{Y})
$$

$\Rightarrow \nabla_{\xi} \nabla_{\xi} \mathrm{X}=\mathrm{X}$, for all $\mathrm{Y}$.

If the soliton is gradient Ricci soliton then $X=\nabla f$, for some smooth function $\mathrm{f}$. So for gradient Ricci soliton we have

$$
\nabla_{\xi} \nabla_{\xi} \nabla \mathrm{f}=\nabla \mathrm{f} .
$$

From (3.6), we have $\nabla_{\xi}\left(g\left(\nabla_{\xi} X, \xi\right)\right)=0$.

$$
\Rightarrow \mathrm{g}\left(\nabla_{\xi} \nabla_{\xi} \mathrm{X}, \xi\right)+\mathrm{g}\left(\nabla_{\xi} \mathrm{X}, \nabla_{\xi} \xi\right)=0 .
$$

$\Rightarrow \mathrm{g}\left(\nabla_{\xi} \nabla_{\xi} \mathrm{X}, \xi\right)=0$, since $\xi$ is a Killing vector field.

Putting $X=\nabla f$ and using (3.12), we have

$$
g(\nabla f, \xi)=0 .
$$

So we can conclude that either $\nabla f$ is orthogonal to $\xi$ or $f$ is a constant function. This completes the proof.

\section{References}

[1]. R. Hamilton, Three manifold with positive Ricci curvature, J. Differential Geom.17(2),(1982),256-306

[2]. R. Hamilton, Four - manifolds with positive curvature operator, J.Differential Geom. 24(2),(1986),153-179.

[3]. G. Perelman, The entropy formula for the Ricci flow and its geometric applications, arxiv.org/abs/math/0211159v1

[4]. G. Perelman, Ricci flow with surgery on three manifolds, arxiv.org/abs/math/0303109.

[5]. R. Hamilton, The Ricci flow on surfaces, Mathematics and general relativity, Contemp. math., 71,(1988),237-261.

[6]. B. Barua, U. C. De, Characterizations of a Riemannian manifold admitting Ricci solitons, Facta Universitatis (Nis), Ser. Math. Inform., 28(2013), no. 2, 127-132.

[7]. U. C. De, M. Turan, A. Yildiz, A. De, Ricci solitons and gradient Ricci solitons on 3-dimensional normal almost contact metric manifolds, Publ. Math. Debrecen, 80(2012), no. 1-2,127-142.

[8]. H. D. Cao, Recent progress on Ricci solitons, arxiv.org/abs/0908.2006.

[9]. H. D. Cao, Geometry of Ricci soliton,Chin.Ann.Math,27B,(2006),121-142. 
[10]. A. Chau, Oliver and C.Schnürer, Stability of gradient Kähler-Ricci solitons,Comm. Anal. Geom.(2005),769-800.

[11]. S.C. Chu, Geometry of 3-Dimensional Gradient Ricci Solitons with Positive Curvature Comm. Anal. Geom.(2005),vol.13(1),129-150.

[12]. A.S. Dancer and M.K.Wang, Some new examples of non-Kḧler Ricci solitons,Math. Res. Lett.16(2009),no. 2,349-363.

[13]. A. Derdzinski, A Myers-type theorem and compact Ricci solitons, Proc. Amer. Math. Soc. 134(2006),3645-3648.

[14]. T. Ivey, Ricci solitons on compact three-manifolds, Differential Geom. Appl. 3(1993), no. 4,301-307.

[15]. D.E. Blair, Contact Manifolds in Riemannian Geometry ,Springer, 1976.

[16]. U. C. De, Ricci solitons and gradient Ricci solitons in a P-Sasakian manifold, The Aligarh Bull. of Maths., 29(2010), no. 1,29-33

[17]. Gurupadavva Ingalahalli, C. S. Bagewadi, Ricci Solitons in alpha-Sasakian Manifolds, ISRN Geometry, Volume 2012(2012), Article ID 421384,13 pages. doi:10.5402/2012/421384.

[18]. C. S. Bagewadi, Gurupadavva Ingalahalli, Ricci Solitons in Lorentzian $\alpha$-Sasakian Manifolds, Acta Mathematica Academiae Paedagogicae Nyregyhaziensis, 28(2012), no. 1,59-68.

[19]. Srabani Debnath, Arindam Bhattacharyya, Second order parallel tensor in trans-Sasakian manifolds and connection with Ricci soliton, Lobachevskii J. Math., 33(2012), no. 4,312-316.

[20]. U. C. De, A. K. Mondal, 3-dimensional quasi-Sasakian manifolds and Ricci solitons, SUT J. Math., 48(2012), no. 1,71-81.

[21]. C. S. Bagewadi, Gurupadavva Ingalahalli, Certain Results on Ricci Solitons in Trans-Sasakian Manifolds, Journal of Mathematics,2013, Article ID 787408, 10 pages.http://dx.doi.org/10.1155/2013/787408

[22]. Chenxu and Meng, Ricci solitons on Sasakian manifolds, arxiv.org/abs/1109.4407.

[23]. U. C. De, A. K. Mondal, The structure of some classes of 3-dimensional normal almost contact metric manifolds, Bull. Malays. Math. Sci. Soc., 36(2013), no. 2,501-509.

[24]. Z. Olszak, The Schouten-van Kampen affine connection adapted to an almost (para) contact metric structure, Publ. Inst. Math. (Beograd) (N. S.), 94(108)(2013),31-42.

[25]. C. Calin and M. Crasmareanu, From the Eisenhart problem to Ricci solitons in f-Kenmotsu manifolds, Bull. Malays. Math. Sci. Soc., 33(2010), no. 3,361-368.

[26]. K. Kenmotsu, A class of almost contact Riemannian manifolds, Tohoku Math. J.,24(1972),93-103.

[27]. C. S. Bagewadi, Gurupadavva Ingalahalli, S. R. Ashoka, A study on Ricci solitons in Kenmotsu manifolds, ISRN Geometry, Volume 2013(2013), Article ID 412593,10 pages.

[28]. A. Yildiz, U. C. De, M. Turan, On 3-dimensional f-Kenmotsu manifolds and Ricci solitons, Ukrainian Math. J., 65(2013), no. 5,684-693.

[29]. G. Nagaraja, C. R. Premalatha, Ricci solitons in f-Kenmotsu manifolds and 3-dimensional trans-Sasakian manifolds, Progress in Applied Mathematics, 3(2012), no. 2,1-6. 